\section{Frakturrisiko: Vitamin D reicht nicht}

Dass Vitamin-D-Mangel das Frakturrisiko bei älteren Menschen erhöht, zeigt die Praxis. Senken lässt sich selbiges durch Vitamin-D-Gabe allerdings nicht. Erst in Kombination mit Kalzium sinkt das Frakturrisiko. Vitamin D allein wirkt sich hingegen positiv auf das Sturzrisiko aus, da es die Muskelfunktion fördert. Auch dieser Effekt wird durch zusätzliches Kalzium verstärkt. Für die Frakturprophylaxe ist demnach die Kombination aus Vitamin D und Kalzium zu empfehlen. DIPART-Group, BMJ 2010, 340:b5463

\section{Medizinstudium auch ohne Abi}

Ärzte in Niedersachsen müssen sich bald auf Konkurrenz vonseiten ihrer Helferinnen einstellen. Denn wer eine abgeschlossene Berufsausbildung und mindestens drei Jahre Berufserfahrung mitbringt, kann in dem Bundesland auch ohne Abitur fachbezogen studieren. Möglich macht dies eine Änderung des Hochschulgesetzes im Rahmen der „offenen Hochschule“. Für die Zulassung zum Medizinstudium ist dann auch beruflich erworbene Kompetenz entscheidend.

Pressemitteilung MWK Niedersachsen

\section{Schneeschippen ist nichts für alte Leute}

Raten Sie Ihren älteren Patienten: Hände weg von der Schneeschippe! Oder zumindest nur gut vermummt in der Kälte arbeiten. Denn Anstrengung bei Minusgraden ist gerade für herzkranke Menschen lebensbedrohlich; es drohen Herzrhythmusstörungen oder Kammerflimmern. Durch physiologische Vorgänge als Reaktion auf die Kälte steigen Blutdruck, Herzfrequenz und Gefäßwiderstand, der Sauerstoffverbrauch des Herzmuskels erhöht sich.

Ärzte Zeitung, 16.01.2010

\title{
Lauftest verblüfft
}

\section{Joggingschuh schlimmer als High Heels}

Mit den neuesten Laufschuhen gelenkschonend Sport treiben? Weit gefehlt! Moderne Joggingschuhe stabilisieren zwar den Fuß, doch Knie- und Hüftgelenk werden dafür umso stärker belastet.

Des einen Freud ist des anderen Leid: In diesem Fall ist die Rede von Fuß-, Knie- und Hüftgelenken. Denn modernes Schuhwerk beim Joggen bettet zwar die Füße optimal, aber die weiter oben liegenden Gelenke haben wenig Nutzen vom neumodischen Fußkleid, so das Ergebniss einer Studie aus Seattle: 68 Frauen und Männer im Alter von 20 bis 50 Jahren unterzogen sich einer „Laufbandanalyse“, bei der sie auf einem Hometrainer einmal mit und einmal ohne Schuhe joggten. Das Ergebnis war ebenso überraschend wie aufschlussreich: Beim Laufen mit speziellen Joggingschuhen wurden Hüfte und Knie deutlich stärker belastet als beim Barfußlauf. Verantwortlich dafür sind nach Meinung der Forscher vor allem der erhöhte Fersenbereich und die zusätzlichen Verstärkungen der Sohle im Mittelfußbereich des Turnschuhs.

Dadurch wird der Bewegungsablauf derart verändert, dass Hüfte und Knie Schaden nehmen können. Bemerkenswerterweise war die Belastung sogar größer als beim Tragen von High Heels! Als Konsequenz sollten sich die Entwickler von Joggingschuhen künftig mehr am Barfußlaufen orientieren.

$(c d)$

\section{CME-Punkte kostenlos in jeder Ausgabe}

\section{CME hilft Ihnen, Ihr Punktekonto zu füllen!}

Mit unseren drei zertifizierten Fortbildungen können Sie pro Heft auf CME.springer.de/CME kostenlos 9 Punkte sammeln. Und so geht's:

\section{Kostenlos teilnehmen in vier Schritten}

\section{Auswählen}

Wählen Sie online auf CME.springer. de/CME den Link zum aktuellen Heft aus. Entscheiden Sie nun, welche Fortbildungseinheiten Sie zum Erwerb von CME-Punkten nutzen wollen. Die Teilnahme an den drei Einheiten der aktuellen Ausgabe ist kostenlos.

\section{Registrieren/Anmelden} Sind Sie bereits bei CME.springer.de registriert? Dann genügt zur Anmeldung die Angabe Ihrer persönlichen Zugangsdaten. Falls Sie zum ersten Mal teilnehmen, bitten wir Sie, sich einmalig zu registrieren. Wir senden Ihnen dann per E-Mail Ihre persönlichen Zugangsdaten zu. Bitte benutzen Sie diese für alle weiteren Teilnahmen zur Anmeldung (Login).

\section{Teilnehmen}

Sie können die elektronische Version der Fortbildung online lesen, ausdrucken oder direkt die 10 CME-Fragen beantworten. Die kostenlose Teilnahme ist 3 Monate nach Erscheinen der Zeitschrift möglich.

\section{CME-Punkte sammeln} Nach erfolgreicher Beantwortung von 7 der 10 CME-Fragen senden wir Ihnen Ihre Teilnahmebestätigung samt Punkten umgehend per E-Mail zu. Diese können Sie bei Ihrer zuständigen Landesärztekammer einreichen. 